\title{
Detecting breath and skin emitted tracers of humans with flame-made sensor arrays
}

\author{
Andreas T. Güntner ${ }^{1}$, Nicolay J. Pineau ${ }^{1}$, Pawel Mochalski', Helmut Wiesenhofer ${ }^{2}$, Agapios Agapiou ${ }^{3}$, \\ Christopher A. Mayhew ${ }^{2}$, Sotiris E. Pratsinis ${ }^{1}$ \\ ${ }^{1}$ Particle Technology Laboratory, ETH Zurich, Sonneggstrasse 3, 8006 Zürich, Switzerland \\ ${ }^{2}$ Breath Research Institute, University Innsbruck, 6850 Dornbirn, Austria \\ ${ }^{3}$ Department of Chemistry, University of Cyprus, 1678 Nicosia, Cyprus \\ pineaun@ptl.mavt.ethz.ch
}

\begin{abstract}
:
Earthquakes are lethal natural disasters frequently burying people alive under collapsed buildings. Tracking entrapped humans from their unique volatile chemical signature with hand-held devices would accelerate urban search and rescue (USaR) efforts. Here, a compact and orthogonal sensor array has been designed to detect the breath- and skin-emitted metabolic tracers acetone, ammonia, isoprene, $\mathrm{CO}_{2}$ and $\mathrm{RH}$, all together serving as sign of life. It consists of three nanostructured metaloxide sensors ( $\mathrm{Si}$-doped $\mathrm{WO}_{3}$, Si-doped $\mathrm{MoO}_{3}$ and Ti-doped $\mathrm{ZnO}$ ), each specifically tailored at the nanoscale for highly sensitive and selective tracer detection along with commercial $\mathrm{CO}_{2}$ and humidity sensors. When tested on humans enclosed in plethysmography chambers to simulate entrapment, this sensor array rapidly detects tracers of human presence with low parts-per-billion ( $p p b)$ level accuracy and precision, unprecedented by portable detectors but required for USaR. These results were validated by bench-top selective reagent ionization time-of-flight mass spectrometry (SRI-TOFMS). As a result, an inexpensive sensor array is presented that can be integrated readily into handheld or even drone-carried detectors for first responders to rapidly screen affected terrain.
\end{abstract}

Key words: Earthquakes, search and rescue, orthogonal sensor array, metabolic tracer, gas sensors

Earthquakes caused more than 780'000 deaths in the last decade and alarmingly, some of the most populous cities are located on fault-lines (e.g. Tokyo, Los Angeles or Delhi). Following an earthquake, many victims are entrapped under collapsed buildings and need help within the first hours. Indispensable for urban search and rescue (USaR) are dogs with their superior ability to sniff entrapped humans from their scent. However, their availability and operational time are limited and they are rather stress-sensitive. Nowadays, specialized equipment is also available to support USaR teams, but these rely only on optical and acoustic probes, not suitable for rapid sweeping of large areas and failing in frequent cases of limited visible access or unconscious victims.

Chemical recognition of the unique volatile signature of humans could improve USaR tools by adding a "third sense", similar to the canines sophisticated nose. Particularly promising to serve as robust sign of life is the combined detection of breath- and skin- emitted metabolic tracers like acetone, ammonia and isoprene originating from lipolysis, protein metabolism and cholesterol biosynthesis, respectively.

Here, we present a novel sensor array based on distinctly selective gas sensors for rapid tracking of entrapped humans (Fig.1) [1]. It consists of three tailor-made gas sensors i.e. Si-doped $\mathrm{WO}_{3}$, Si-doped $\mathrm{MoO}_{3}$ or Ti-doped $\mathrm{ZnO}$ featuring high sensitivities and selectivities to the metabolic tracers acetone [2], isoprene [3] and ammonia [4], respectively (Fig. 1b). These sensors consist of nanostructured, highly porous metal-oxide films (Fig. 1d) that offer high surface area to detect tracers even at the lowest ppb concentrations. Such films are obtained by direct deposition of flame-made nanoparticles on substrates forming finely structured sensing networks, as shown exemplarily for Ti-doped $\mathrm{ZnO}$ (Fig. 1e). Combined with commercial $\mathrm{CO}_{2}$ and $\mathrm{RH}$ sensors, they result in an array with nearly orthogonal sensing characteristics enabling superior discrimination power and robustness to accurately detect the chemical signature of humans. 

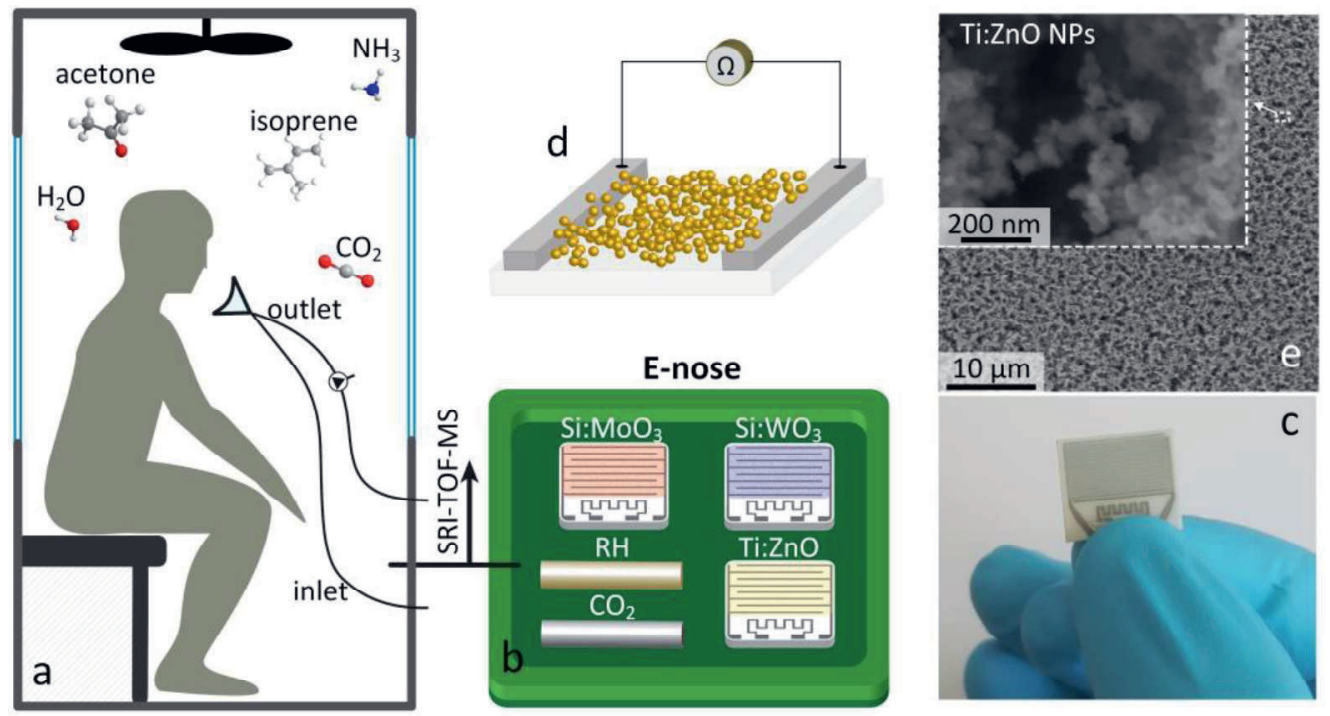

Fig. 1. a) Skin- \& breath-borne volatiles of entrapped volunteers accumulate in a plethysmographic chamber. b) The sensor array consists of three chemoresistive sensors, Si-doped $\mathrm{WO}_{3}$, Ti-doped $\mathrm{ZnO}$ and Si-doped $\mathrm{MoO}_{3}$, to monitor acetone, isoprene and ammonia, respectively, together with commercial $\mathrm{CO}_{2}$ and humidity. c) Image of a single sensor. d) Nanoparticles form a porous sensing network as shown by (e) scanning electron microscopy. [1]

We applied the sensor array to detect and monitor the volatile chemical signature of humans. Therefore, nine volunteers were enclosed individually in plethysmographic chambers (Fig. 1a) to mimic entrapment conditions. The testing course for each volunteer lasted for $120 \mathrm{~min}$ with skin $(0-60$ min) and skin + breath $(60-120 \mathrm{~min})$ emissions into the chamber being investigated separately. Fig. 2 shows the corresponding sensor array estimated acetone (a), isoprene (b), ammonia (c), $\mathrm{RH}$ (d) and $\mathrm{CO}_{2}$ (e) concentration profiles of five volunteers when measured every $20 \mathrm{~min}$. Note that the data of the other four randomly selected volunteers were used for "training" of a multivariate linear regression model

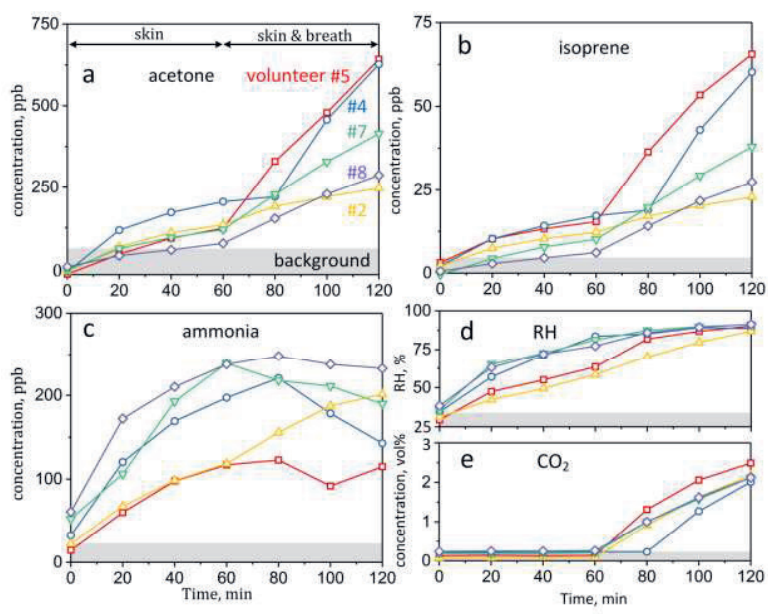

Fig. 2. Sensor array monitored acetone (a), isoprene (b), ammonia (c), $\mathrm{RH}(d)$ and $\mathrm{CO}_{2}(e)$ concentrations of five volunteers as a function of entrapment time. In case of volunteer \#4, skin (only) emissions lasted accidentally for $80 \mathrm{~min}$. Room air (background) concentrations are indicated in grey. Adopted from [1].
Typically, acetone, isoprene and $\mathrm{CO}_{2}$ change only little during skin emission while they increase significantly when also exhaled, as detected by the sensor array and consistent with SRI-TOF-MS analysis. As a result, these tracers indicate human presence during combined emissions rather early as their concentrations rapidly exceed background levels. On the other hand, ammonia and RH increase significantly during skin emission and differ from the background even after short entrapment. Later, both tend to level off and ammonia may even decrease in some cases, as confirmed by SRI-TOF-MS.

In conclusion, the detector recognizes human presence robustly due to multi-tracer assessment already after very short entrapment time. Furthermore, the sensor array can be incorporated easily into portable detectors to support first responders to find trapped victims after earthquakes.

\section{References}

[1] Güntner, A. T.; Pineau, N. J.; Mochalski, P.; Wiesenhofer, H.; Agapiou, A.; Mayhew, C. A.; Sotiris, E. P., Sniffing Entrapped Humans from their Chemical Signature. (in review), (2018).

[2] Righettoni, M.; Tricoli, A.; Gass, S.; Schmid, A.; Amann, A.; Pratsinis, S. E., Breath acetone monitoring by portable $\mathrm{Si}: \mathrm{WO}_{3}$ gas sensors. Anal Chim Acta, 69-75 (2012).

10.1016/j.aca.2012.06.002.

[3] Güntner, A. T.; Pineau, N. J.; Chie, D.; Krumeich, F.; Pratsinis, S. E., Selective sensing of isoprene by Ti-doped $\mathrm{ZnO}$ for breath diagnostics. J. Mater. Chem. B 32, 5358-5366 (2016); 10.1039/C6TB01335J.

[4] Güntner, A. T.; Righettoni, M.; Pratsinis, S. E., Selective sensing of $\mathrm{NH}_{3}$ by Si-doped $\alpha-\mathrm{MoO}_{3}$ for breath analysis. Sensor Actuat B-Chem, 266-273 (2016); 10.1016/j.snb.2015.09.094. 